\title{
A type of incompatibility between genes and their genetic background inducing decrease in heterozygote viability approximately equal to that of homozygotes, found in a natural population of Drosophila
}

\author{
KENJI YUKUHIRO* \& TERUMI MUKAI† \\ Department of Insect Genetics and Breeding, National Institute of Sericultural and Entomological Science, Tsukuba, \\ Ibaraki 305 and tDepartment of Biology, Faculty of Science, Kyushu University, Fukuoka 812, Japan
}

\begin{abstract}
In the Osaka population of Drosophila melanogaster, we found an incompatibility between second chromosomes and their genetic backgrounds, where a decrease in the average viability of heterozygotes in the foreign genetic background relative to that of the native one $\left(A^{\prime} / A=0.889\right)$ was about equal to that in non-lethal homozygotes $\left(C^{\prime} / C=0.874\right)$. This feature is different from that of the incompatibility found in the Ishigakijima population, where little difference in average viabilities of heterozygotes between the native and foreign backgrounds was found, whereas in mean non-lethal homozygotes viabilities a large decrease was seen. This feature is supposed to be induced by the $P-M$ hybrid dysgenesis, as strong $P$-transposase activity was shown in this population with the GD sterility test. Although a property similar to that of the Osaka population had been detected in the Katsunuma population, an increased frequency of the lethal-carrying chromosomes had been found in the foreign genetic background relative to the native one, and was not seen in the Osaka population.
\end{abstract}

Keywords: D. melanogaster, genetic loads, incompatibility, local population, $P$-transposase activity, viability.

\section{Introduction}

There are many examples showing that interaction or incompatibility between genes and their genetic backgrounds results in deleterious effects on fitness in Drosophila (see Woodruff et al., 1983). P elementinduced hybrid dysgenesis of D. melanogaster (Kidwell et al., 1977) may be taken as an example of such incompatibilities, and the frequent occurrence of deleterious mutations and sterility is observed in the germline of $F_{1}$ progeny from specific crosses (see Engels, 1989). The $P$ element more often tends to induce mutations that cause a large decrease in viability (lethal and semi-lethal) than mutations that have mildly deleterious effects (Yukuhiro et al., 1985). For example, the incompatibility between second chromosomes and genetic backgrounds observed in the Ishigakijima population of $D$. melanogaster is probably

*Correspondence.

†Died in April 1990. caused by the harmful effects of $P$-induced mutations (Suh \& Mukai, 1991).

However, there are examples of incompatibilities that are difficult to explain by the harmful effects of $P$ induced hybrid dysgenesis, such as delta (Minamori, 1969a,b). Furthermore, agents other than transposable elements have been proposed to cause incompatibilities that result in detrimental effects on the fitness of an organism in one of the reciprocal crosses (e.g. in $D$. simulans; Hoffmann et al., 1986): the causal agents are rickettsia-like microorganisms collectively classified in the genus Wolbachia (Weiss \& Chang, 1984), which induce a high rate of embryonic mortality in crosses between strains carrying different kinds of cytoplasm. O'Neill \& Karr (1990) indicated the existence of two different types of Wolbachia based on the bidirectional incompatibility between $D$. simulans strains from different geographical origins.

In this paper, we describe a type of incompatibility that occurs between a second chromosome from the 
Osaka population and a particular genetic background from a laboratory stock of $D$. melanogaster. In this situation, we have found a decrease in viability relative to the homologous genetic background. The amount of difference in average viabilities of heterozygotes between the two genetic backgrounds was approximately equal to that of the homozygotes. We have compared these results with those of the Katsunuma and Ishigakijima populations (Mukai et al., 1985; Suh \& Mukai, 1991, respectively). The present case of incompatibility has not been attributable to any particular causal elements.

\section{Materials and methods}

\section{Stocks}

The following stocks were used in the present studies.

$O S$-84IF. A series of isofemale lines of D. melanogaster, collectively called OS-84IF, which originated from the flies collected at Komagatani, Habikino City, Osaka Prefecture in August 1984, was maintained as isofemale lines at $18^{\circ} \mathrm{C}$ in the laboratory of Population Genetics, Kyushu University.

$C-160$. A balancer stock of $\operatorname{In}(2 L R) S M 1$ and $\operatorname{In}(2 L R) b w^{V I}$, which is abbreviated as $C y / P m$. This strain is a typical $M$ strain.

$C-160-N$. The genotypes of the second chromosomes are the same as those of $\mathrm{C}-160$, but other chromosomes and the cytoplasm are replaced with repeated back crosses by the flies (OS-24-I) of the Osaka population sampled in 1981. Thus, the cytotype of this stock is the $P$ according to our previous test.

$\pi_{2}$. A typical $P$ strain, which originated from the Madison, WI population (Engels \& Preston, 1979).

Canton $S$. A standard laboratory wild-type stock known to be a typical $M$ strain.

$y s n^{w} / y^{+} Y ; b w ; s t$. This is a pseudo $M$ strain with a few defective $P$ elements. The $s n^{w}$ allele was caused by two $P$ element insertions in the region upstream of the singed locus (Engels, 1983).

$C(1) D X, y w f ; Y / F M 7$. A strain in which females have the attached-X chromosomes homozygous for the recessive markers $y, w$ and $f$. Males carry $F M 7$ balancer X chromosome.

\section{Extraction of second chromosomes}

Two hundred and ninety-nine second chromosomes were randomly extracted using the $C y / P m$ method with C-160 from isofemale lines (OS-84IF). They were established in the foreign genetic background that is originated from the C-160 stock, 'foreign' to the Osaka population (Fig. 1).

With the same mating scheme using C-160-N instead of $\mathrm{C}-160$, the same number of second chromosome lines was independently established from OS84IF in the native genetic background (Fig. 1).

Using this mating scheme, if mutations were induced through this extraction process by hybrid dysgenesislike events, they are segregated in a line. Accordingly, our estimates of viabilities may be affected by the harmful effects of such mutations in a segregated condition.

\section{Estimation of viability}

The $C y$ method of Wallace (1956) was performed to estimate homozygous and heterozygous viabilities (see Mukai et al., 1985, for detail)

Viability is estimated as follows (Wallace, 1956):

$V=\frac{2 \times(\text { number of wild-type flies })}{\text { number of } C y \text { flies }+1}$

\section{Estimation of genetic load}

Homozygous loads relative to the average viability of random heterozygotes were estimated using the method of Greenberg \& Crow (1960). Let $A, B$ and $C$ be the average viabilities of random heterozygotes, all homozygotes, and lethal-free homozygotes, respectively. In the present studies, we defined the line of which viability index was estimated to be less than 0.1 as a lethal line. Then, the total load $(T)$ can be estimated and partitioned into the detrimental $(D)$ and lethal $(L)$ loads by the following formulae:

$\left.\begin{array}{l}T=\ln A-\ln B \\ D=\ln A-\ln C \\ L=\ln C-\operatorname{In} B\end{array}\right\}$

\section{Gonadal dysgenic sterility test}

Following the method of Engels \& Preston (1979), we determined the cytotypes and the genotypes (i.e. the $P$ transposase activity) of OS-84IF lines by means of $A^{*}$, $B^{*}, A$ and $B$. In this survey, Canton $S$ and $\pi_{2}$ were used as typical $M$ and $P$ strains, respectively (see Yukuhiro $\&$ Mukai, 1986 for details). 


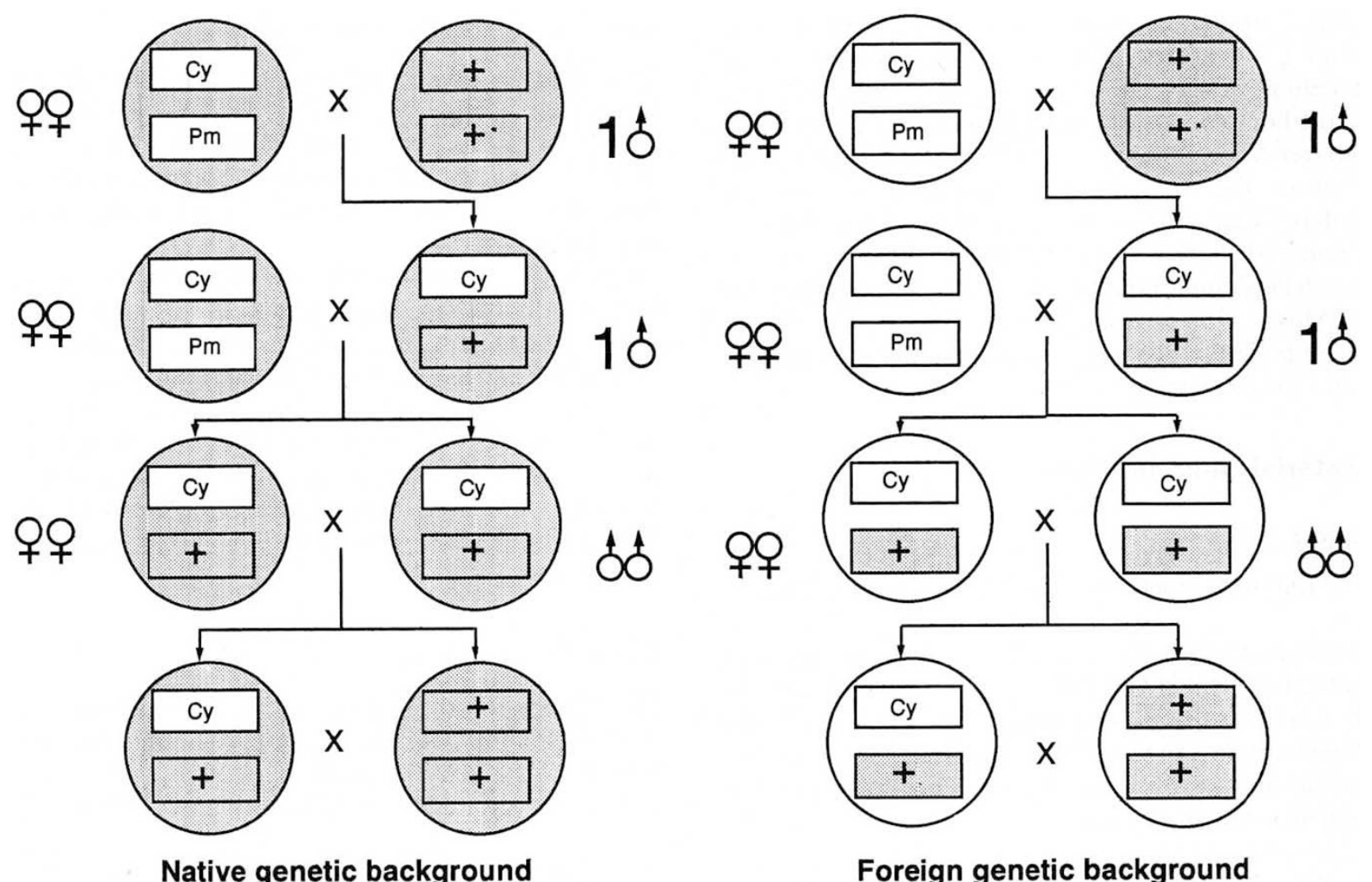

Fig. 1 Mating scheme for extraction of second chromosomes using two types of $C y / P m$ strains.

\section{$s n^{w}$ hypermutability test for autosomes and $Y$ chromosome}

A single male from each of the isofemale lines sampled from OS-84IF was crossed to females homozygous for $y s n^{w} ; b w$; st to obtain sons whose mutability was to be measured (Engels, 1983). A single son from each cross was mated to five $C(1) D X y w f / Y$ females. Four days after the crosses were made, all flies were transferred to the second vial. After four days, all parental flies were discarded. Male progeny were scored for their bristle phenotype (i.e. the extreme phenotype, $s n^{\mathrm{e}}$, the nearly wild-type, $s n^{+}$, and the parental type, $s n^{\mathrm{w}}$ ) until the 17 th day.

As the control, the same kinds of crosses were made using $\pi_{2}$ instead of wild-type flies.

All the experiments, except a series of crosses, $A^{*}$, $B^{*}, A$ and $B$, made in the gonadal dysgenic sterility test, were performed at $25^{\circ} \mathrm{C}$.

\section{Results}

\section{Estimations of viabilities and genetic loads}

To confirm the incompatibility between second chromosomes and genetic backgrounds, we estimated homozygote and heterozygote viabilities of two types of second chromosome lines, which were established in different genetic backgrounds.

In the native background, the frequency of lethalcarrying chromosomes was $80 / 299$ or $0.268 \pm 0.026$. The estimated total load $(T)$, detrimental load $(D)$ and lethal load $(L)$ were $0.628,0.320$, and 0.308 , respectively, where $A=1.1594 \pm 0.0072$, $B=0.6188 \pm 0.0242$ and $C=0.8418 \pm 0.0156$ (Fig. 2). The $D / L$ ratio was 1.04 .

In the foreign background, the frequency of lethal carrying chromosomes was $72 / 299$ or $0.241 \pm 0.025$, which was not significantly different from that in the native background $\left(\chi_{\mathrm{d} . \mathrm{f}=1}^{2}=0.565,0.30<P<0.40\right)$. However, the average viability of heterozygotes $\left(A^{\prime}\right)$ was $1.0307 \pm 0.0066$, which was significantly lower than that in the native background $(A)\left(t_{\text {d.f. }=581}\right.$ $=13.13, P<0.0005)$. The average viabilities of all homozygotes $\left(B^{\prime}\right)$ and non-lethal homozygotes $\left(C^{\prime}\right)$ were $0.5625 \pm 0.0208$ and $0.7359 \pm 0.0141$, respectively. The $B^{\prime}$ value was significantly lower than the $B$ value at the $5 \%$ level $\left(t_{\text {d.f. }=596}=1.75\right)$, and the $C^{\prime}$ value was significantly lower than the $C$ value at the $0.05 \%$ level $\left(t_{\text {d.f. }=444}=4.93\right)$. However, the estimated genetic loads were not significantly different. The estimated genetic loads, $T^{\prime}, D^{\prime}$ and $L^{\prime}$ were $0.606,0.337$ 

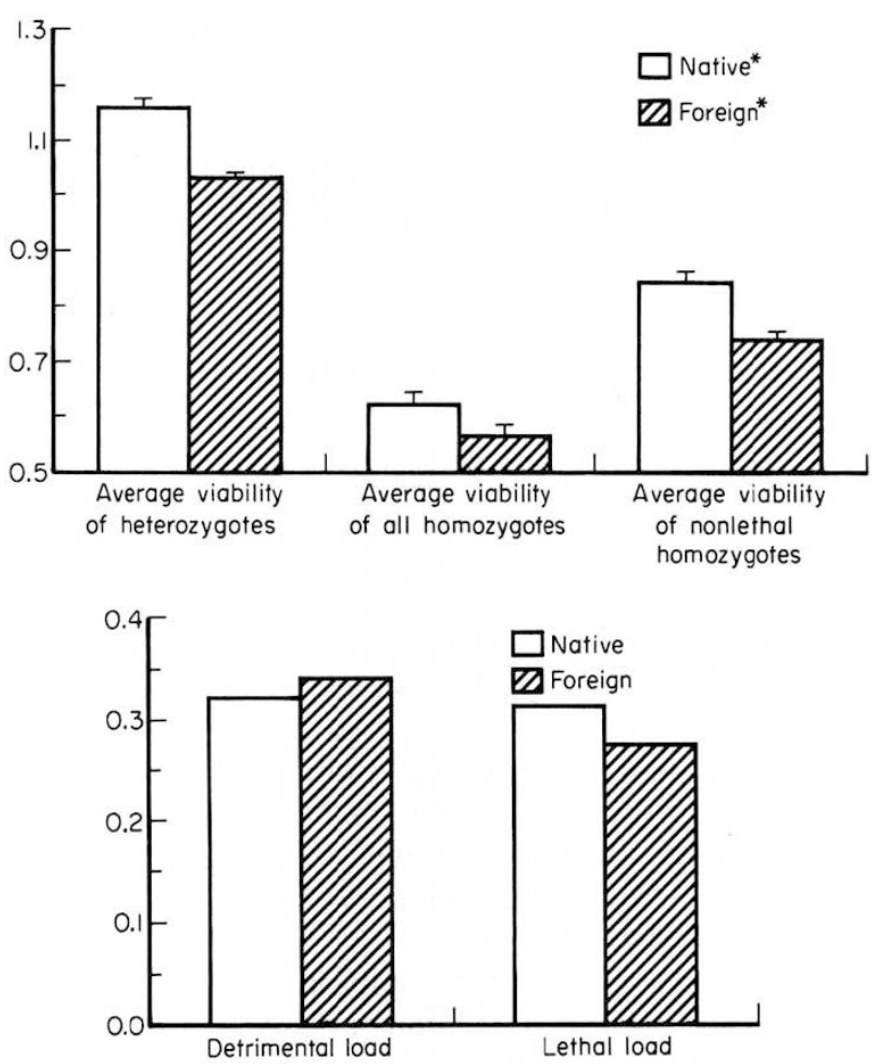

and 0.269 , respectively. The $D^{\prime} / L^{\prime}$ ratio was 1.25 . These results are also shown in Fig. 2.

\section{Evaluation of $P$-transposase activity}

We conducted two types of tests for assessing $P$ transposase activity to investigate the influence of $P$ element activity on the decrease in viabilities in the foreign genetic background.

Firstly, we examined GD sterility. One hundred and fifty-eight isofemale lines were chosen from OS-84IF for both determinations of cytotype and the genotype. However, a total of 155 isofemale lines was used in cross $A^{*}$ and 153 in cross $B^{*}$ because of some unsuccessful matings. In the determination of the genotype, one line was missed in cross $A$. So one to three females (cross $A^{*}$ and cross $B$ ) or males (cross $A$ and cross $B^{*}$ ) from each isofemale line were used for crosses. From each cross, 9-16 daughters were sampled and their sterility was individually tested as described in Engels \& Preston (1979).

As we detected frequencies of sterile daughters estimated in crosses $B^{*}$ and $B$ of less than 20 per cent in these studies, we used the ' 20 per cent criterion' for determination of cytotype and the genotype according to Yukuhiro \& Mukai (1986): when a fraction of sterile

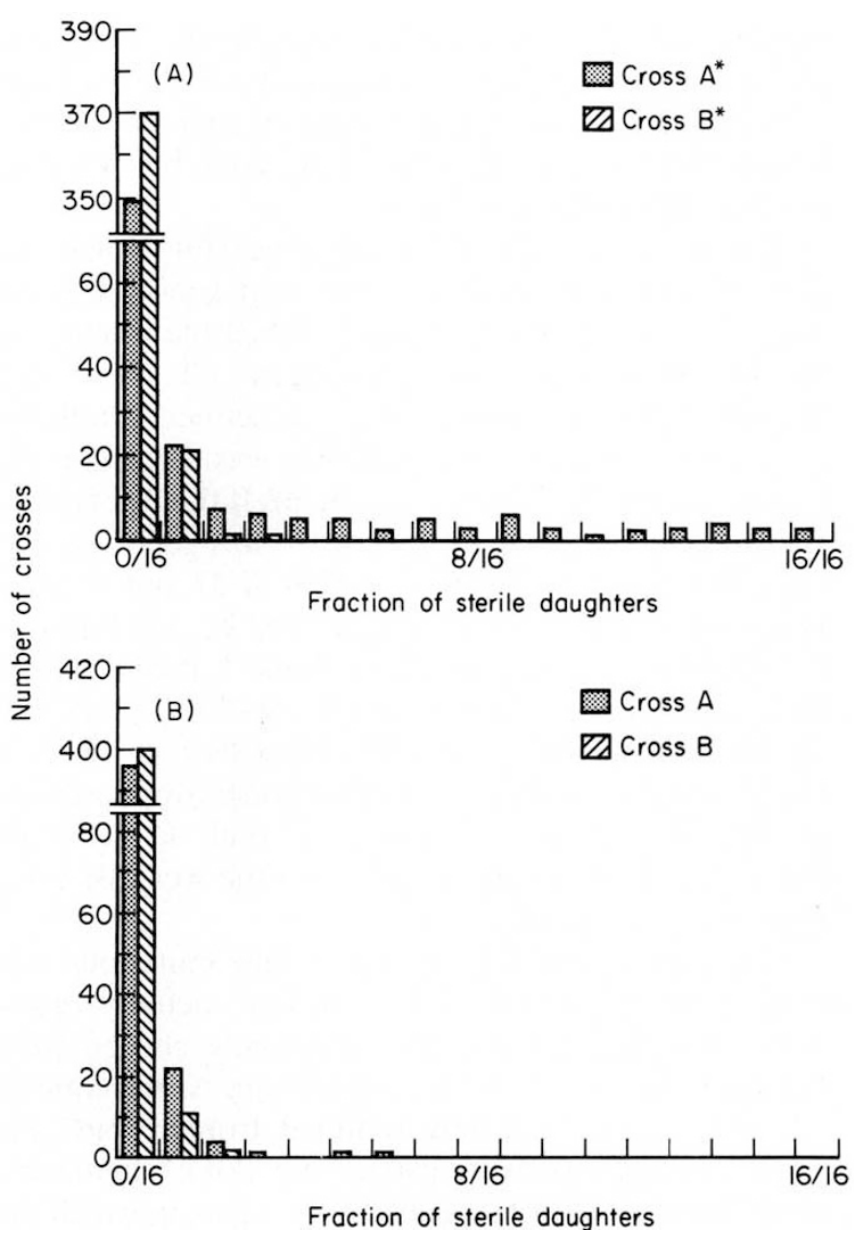

Fig. 3 (a) Distributions of fractions of sterile daughters in the determination of the cytotype. (b) Distributions of fractions of sterile daughters in the determination of the genotype.

daughters of cross $A^{*}$ was more than 20 per cent, the cytotype of their female parent was classified as $M$. In any other case, the cytotype of their parent was defined as $P$ (Yukuhiro \& Mukai 1986).

There were 45 of 429 females in cross $A^{*}$ classified as an $M$ cytotype-carrying female. The results are shown graphically in Fig. 3(a). On the basis of individual isofemale lines, 121 isofemale lines of 155 carried the $P$ cytotype; in 32 lines, $P$ and $M$ segregated, and only two lines carried the $M$ cytotype.

In the determination of the genotype, when the fraction of sterile daughters was more than 20 per cent, we defined the genotype of the parent male as $P$.

On the basis of individual parental males, two of 432 males were classified as the $P$ genotype (Fig. 3(b)). These two $P$ genotype males were from different isofemale lines. The results of the determination of the genotype on the isofemale line basis are summarized as follows: 155 of 157 were determined as not $P$ geno- 
type. In two of 157 isofemale lines, individuals of the $P$ and not $P$ genotypes were segregating, as the fractions of sterile daughters of some males of each of two isofemale lines were more than 20 per cent, but the fractions of the rest were not so.

The results of the isofemale lines for which the determinations of both cytotype and genotype were successful are shown in Table 1. When the fraction of sterile daughters in cross $A$ did not exceed 20 per cent, the genotype of the male parent was defined as follows: if the cytotype of this isofemale line was defined as $M$, this genotype was estimated to be $M$. If the cytotype of the isofemale line was defined as $P$, this genotype was estimated to be $Q$ (Kidwell, 1979). If $M$ and $P$ cytotypes were segregating, the genotype of this line was estimated to be $Q$ and $M$. From Table 1 , it can be seen that 120 of 154 isofemale lines were of the $Q$ type; in 30 lines, the $Q$ and $M$ type flies coexisted; in one line, the $P$ and $Q$ type flies were segregating; two lines were of the $M$ type. In one line, the weak $P$ genotype individuals and individuals carrying the weak $M$ cytotype were segregating.

Secondly, a test for $s n^{w}$ secondary mutations was performed to assess $P$-transposase activity more accurately. Seventy-one lines randomly chosen from OS-84IF were used for this experiment. As the control, 71 males were randomly sampled from $\pi_{2}$ and the same kinds of tests were carried out. All of them were determined as the $Q$ type except one line, in which the $P$ and $Q$ flies were segregating in the GD sterility. Three sets of experiments were performed. Averaging estimates of respective crosses of the control, the average rate of $s n^{w}$ secondary mutation was calculated to be $0.3887 \pm 0.0129$. The distributions of estimated mutation rates of the control and experimental lines are shown in Table 2.

When all the estimates of the $s n^{w}$ secondary mutation rate in the experimental crosses were averaged, the

Table 1 Results of test for $\mathrm{F}_{1}$ female sterility in the $P-M$ hybrid dysgenesis system: classification of isofemale lines sampled from OS-84IF for genotype and cytotype

\begin{tabular}{llccl}
\hline & \multicolumn{2}{l}{ Cross $A^{*}$} & & \\
\cline { 2 - 5 } & & $\mathrm{F}$ & $\mathrm{F}$ and $\mathrm{S}$ & $\mathrm{S}$ \\
\hline & $\mathrm{F}$ & $120(\mathrm{Q})$ & $30(\mathrm{Q}$ and $\mathrm{M})$ & $2(\mathrm{M})$ \\
Cross $A$ & $\mathrm{~F}$ and $\mathrm{S}$ & $1(\mathrm{P}$ and $\mathrm{Q})$ & $1(\mathrm{P}, \mathrm{Q}$ and $\mathrm{M})$ & 0 \\
& $\mathrm{~S}$ & 0 & 0 & 0 \\
\hline
\end{tabular}

F - 'fertile', S - 'sterile', F and S - 'fertile' and 'sterile' individuals are segregating.

Cross $A^{*}$ : unknown strain $(19) \times \pi_{2}\left(0^{*} 0^{*}\right)$.

Cross $A$ : Canton $S(\%)) \times$ unknown strain (10). mean estimate was $0.0170 \pm 0.0036$. This value was significantly lower than that of the control $\left(t_{\mathrm{d} \text {.f. }=140}=19.99, P<0.0005\right)$.

From these results as a whole, the Osaka population can be described as the $Q$ type population.

\section{Discussion}

In the present studies, we obtained the following results:

1 Homozygote and heterozygote viabilities were decreased in the foreign genetic background compared with those in the native genetic background. The decrease in average heterozygote viability was approximately equal to that of homozygotes $\left(A^{\prime} / A=0.889, C^{\prime}\right)$ $C=0.874)$. Thus, little increase was detected in detrimental load $\left(D^{\prime}: D=0.337: 0.320\right)$.

2 Examining $P-M$ hybrid sterility and $s n^{w}$ hypermuta-bility, it was found that, in the Osaka population, the majority of isofemale lines were classified as $Q$; most of the remaining lines were classified as $M$, while $P$ type lines were very rare.

Some features of the results in (1) are similar to those of Japanese populations previously reported on the genetic background effects (Mukai et al., 1985; Suh \& Mukai, 1991), although there are some differences between them. In the Ishigakijima population, larger differences were found in the viabilities between the two types of genetic background in the homozygotes than in the heterozygotes. The estimates of average viabilities are shown in Table 3 . The ratio between the two average non-lethal viabilities $\left(C^{\prime} / C\right)$ was 0.892 , whereas that between the averages of heterozygote viabilities $\left(A^{\prime} / A\right)$ was 0.976 . As a result, the $D$ value

Table 2 Frequency distribution table of $s n^{w}$ hypermutation rates

\begin{tabular}{lcc}
\hline $\begin{array}{l}\text { Range of } \\
\text { mutation rates }\end{array}$ & $\pi_{2}$ & Osaka \\
\hline $0-0.05$ & 0 & 65 \\
$0.05-0.15$ & 1 & 5 \\
$0.15-0.25$ & 8 & 1 \\
$0.25-0.35$ & 19 & 0 \\
$0.35-0.45$ & 22 & 0 \\
$0.45-0.55$ & 12 & 0 \\
$0.55-0.65$ & 7 & 0 \\
$0.65-0.75$ & 1 & 0 \\
$0.75-0.85$ & 1 & 0 \\
Total & 71 & 0.0170 \\
Average mutation & 0.3887 & \pm 0.0036 \\
\multicolumn{1}{c}{ rate } & \pm 0.0129 & \\
\hline
\end{tabular}


Table 3 Average viabilities in Osaka, Katsunuma and Ishigakijima populations

\begin{tabular}{llrcc}
\hline & & \multicolumn{1}{c}{ Osaka } & Katsunuma $\rceil^{\prime}$ & Ishigakijima \\
\hline A & Native & 1.1594 & 1.1189 & 0.9697 \\
& & \pm 0.0072 & \pm 0.0057 & \pm 0.0039 \\
& Foreign & 1.0307 & 0.9730 & 0.9465 \\
& & \pm 0.0066 & \pm 0.0066 & \pm 0.0029 \\
B & Native & 0.6188 & 0.6762 & 0.6243 \\
& & \pm 0.0242 & \pm 0.0215 & \pm 0.0196 \\
& Foreign & 0.5625 & 0.5286 & 0.5685 \\
& & \pm 0.0208 & \pm 0.0205 & \pm 0.0174 \\
C & Native & 0.8418 & 0.8492 & $0.8107^{*}$ \\
& & \pm 0.0156 & \pm 0.0149 & \\
& Foreign & 0.7359 & 0.7651 & $0.7231^{*}$ \\
& & \pm 0.0141 & \pm 0.0119 & \\
L & Native & 0.268 & 0.176 & 0.235 \\
& & \pm 0.026 & \pm 0.020 & \pm 0.021 \\
& Foreign & 0.241 & 0.278 & 0.218 \\
& & \pm 0.025 & \pm 0.024 & \pm 0.021 \\
\hline
\end{tabular}

†From Mukai et al.(1985).

¥From Suh \& Mukai (1991).

*The standard errors of means of these parameters were not shown in the original paper.

A indicates the average viability of heterozygotes.

$\mathrm{B}$ is the average viability of all homozygotes.

$\mathrm{C}$ means the average viability of lethal free homozygotes.

$\mathrm{L}$ implies the frequency of lethal carrying chromosomes.

became larger in the foreign genetic background than in the native one $(0.269$ vs. 0.179$)$. In the Katsunuma population, however, approximately equal decreases in viabilities were found in non-lethal homozygotes and heterozygotes in the foreign genetic background compared with the native one $\left(C^{\prime} / C=0.901, A^{\prime} / A=\right.$ 0.870 , see Table 3) (Mukai et al., 1985). As a result, little difference was detected between the $D$ values $(0.240$ vs. 0.276$)$. Ratios similar to those in the Katsunuma population could be seen in the Osaka population, as described above.

As the $P$ elements are fairly active in the Ishigakijima population, Suh \& Mukai (1991) consider that deleterious effects on fitness induced by $P-M$ hybrid dysgenesis cause a decrease in viability in the foreign background relative to the native one. Their consideration is based on the result that the $P$ element frequently induces lethal and semi-lethal mutations, with relatively small harmful effects in the heterozygous condition (Yukuhiro et al., 1985).

The average activity of $P$-transposase was estimated to be very weak in the Osaka population, a result similar to that obtained in the Katsunuma population (Mukai et al., 1985). Furthermore, the patterns of decrease in viability detected in the two groups of heterozygotes of the Ishigakijima population were different from those of the Katsunuma and Osaka populations. Thus, the possibility that $P$ elements cause the incompatibilities between second chromosomes and their genetic background in the Katsunuma and Osaka populations seems to be difficult to explain. We may consider some of the candidates causing the incompatibilities found in the Katsunuma and Osaka populations. One possibility is transposable elements other than the $P$ element that can induce mildly deleterious mutations. This idea is based on the observation that the decrease in the mean viability of non-lethal homozygotes was approximately equal to that of heterozygotes, which corresponded to the additive harmful effects of induced mutations. Furthermore, Mukai \& Yukuhiro (1983) reported that mildly deleterious mutations are more frequently induced than the standard rate $(0.14 /$ second chromosome/generation) on a second chromosome from the Osaka population (Mukai, 1964). We also propose cytoplasmic organisms, such as microorganisms, viruses, etc. (see Rousset \& Raymond, 1991; Woodruff et al., 1983). However, we cannot completely reject the possibility that $P$ element derivatives induce these types of incompatibilities.

Although some similarities were seen in the results of the Osaka and Katsunuma populations, the frequencies of lethal chromosomes were different in the two genetic backgrounds of the Katsunuma population, whereas no significant difference was found in the Osaka population (Table 3). This may indicate that the agent inducing incompatibility in the Katsunuma population is not the same as that in the Osaka population.

Incompatibility between genes and genetic background, including hybrid dysgenesis, can play an important role in speciation because the harmful effects on the fitness of the organism can be induced after mating occurs between individuals from different populations. However, such studies are limited to a small number of species. Investigation of these kinds of incompatibilities, especially on the molecular level, may supply valuable information on the mechanism of species divergence, and contribute significantly to ecological studies.

\section{Acknowledgements}

We thank Fuyuo Hihara for supplying the strain, $y s n^{w} /$ $y^{+} Y$; bw; st. We also thank Ko Harada, Toshiyuki S. Takano and Marian R. Goldsmith for their valuable comments on the manuscripts.

\section{References}

ENGELS, W. R. 1983. The $P$ family of transposable elements in Drosophila. Ann. Rev. Genetics, 17, 315-344. 
ENGELS, w. R. 1989. $P$ elements in Drosophila. In: Berg, D. \& Howe, M. (eds) Mobile DNA, ASM Publications, pp. 437-489.

ENGELS, W. R. AND PRESTON, C. R. 1979. Hybrid dysgenesis in Drosophila melanogaster: the biology of female and male sterility. Genetics, 92, 161-174.

GREENBERG, R. AND CROW, J. F. 1960. A comparison of the effect of lethal and detrimental chromosomes from Drosophila populations. Genetics, 45, 1154-1168.

HOFFMANN, A. A., TURELLI, M. AND SIMMONS, G. M. 1986. Unidirectional incompatibility in between populations of Drosophila simulans. Evolution, 40, 692-701.

KIDWELL, M. G. 1979. Hybrid dysgenesis in Drosophila melanogaster: the relationship between the $P-M$ and $I-R$ interaction systems. Genet. Res. Camb., 33, 205-217.

KIDWELL, M. G., KIDWELl, J. F. AND SVED, J. A. 1977. Hybrid dysgenesis in Drosophila melanogaster: a syndrome of aberrant traits including mutations, sterility and male recombinants. Genetics, 83, 813-833.

MINAMORI, s. 1969a. Extrachromosomal element delta in Drosophila melanogaster. I. Gene dependence of killing action and multiplication. Genetics, 62, 583-596.

MINAMORI, s. 1969b. Extrachromosomal element delta in Drosophila melanogaster. II. Transmission through male parent. Jpn. J. Genet., 44, 347-354.

MUKAI, T. 1964. The genetic structure of natural populations of Drosophila melanogaster: I. spontaneous mutation rate of polygenes controlling viability. Genetics, 50, 1-19.

MUKAI, T., BABA, M., AKIYAMA, M., UOWAKI, N. KUSAKABE, S. AND TAJIMA, F. 1985. Rapid change in mutation rate in a local population of Drosophila melanogaster. Proc. Natl. Acad. Sci. U.S.A., 82, 7671-7675.
MUKAI, T. AND YUKUHIRO, K. 1983. An extremely high rate of deleterious viability mutations in Drosophila possibly caused by transposon in non-coding regions. Proc. J. Acad., 59, 316-319.

O'NEILL, S. L. AND KARR, J. L. 1990. Bidirectional incompatibility between conspecific populations of Drosophila simulans. Nature, 348, 178-180.

ROUSSET, F. AND RAYMOND, M. 1991. Cytoplasmic incompatibility in insects: why sterilized females? Trends Ecol. Evol., 6, 54-57.

SUH, D. S. AND MUKAI, T. 1991. The genetic structure of natural populations of Drosophila melanogaster. XXIV. Effects of hybrid dysgenesis on the components of genetic variance of viability. Genetics, 127, 545-552.

WALLACE, B. 1956. Studies on irradiated populations of Drosophila melanogaster. J. Genet., 54, 280-293.

WEISS, E. G. AND CHANG, K-P. 1984. Wolbchieae. In: Krieg, N. R. and Holt, J. H. (eds), Bergey's Manual of Systematic Bacteriology, vol. 1, Williams \& Wilkins, pp. 711-717.

WOODRUFF, R. C., SLATKO, B. E. AND THOMPSON, Jr. J. N. 1983. Factors affecting mutation rate in natural popuations. In: Ashburner, M. Carson, H. L. and Thompson, Jr., J. R. (eds), The Genetics and Biology of Drosophila, Vol. 3c, Academic Press, London, pp. 37-124.

YUKUHIRO, K., HARADA, K. AND MUKAI, T. 1985. Viability mutations induced by the $P$ elements in Drosophila melanogaster. Jpn. J. Genet., 60, 531-537.

YUKUHIRO, K. AND MUKAI, T. 1986. Increased detrimental load possibility caused by a transposon in a local population of Drosophila melanogaster. Jpn. J. Genet., 61, 25-43. 